\title{
Internet-Memes als multimodale Sprechakte in öffentlichen Diskursen anhand von Beispielen aus Antwortthreads von@realDonaldTrump
}

\author{
Andreas Osterroth (Universität Koblenz-Landau)
}

\begin{abstract}
Internet memes are highly relevant for digital communications but there is not much research about their pragmatic uses. Besides being texts, memes are also multimodal speech acts. They are very similar to the speech acts Searle described, but there are some differences. The communicative function of memes is in accord with Keller's principle for social success, which is aimed for by all speakers. It is shown that people on Twitter use memes as speech acts when replying to Donald Trump's Tweets. The difference between meme speech acts and traditional speech acts is their emphasis on the expressive function. While the meme speech acts can take any of Searle's described functions, there is almost always an expressive element to be found.
\end{abstract}

\section{$1 \quad$ Einleitung}

Internet-Memes sind ein relativ neues Phänomen und in der Linguistik noch nicht in aller Breite erforscht. Zwar gibt es erste Annäherungsversuche (cf. Bülow/Johann 2019, Dynel 2016, Krieg-Holz/Bülow 2019, Morger 2017, Osterroth 2015, Shifman 2014), aber eine vollständige Beschreibung steht noch aus. Dieser Beitrag versucht, eine Lücke zu schließen, indem er zeigt, dass Internet-Memes als Sprechakte bzw. Sprache-Bild-Text-Akte im Sinne von Searle verstanden werden können.

John R. Searles Essay (1984 [engl. 1969]) zu den Sprechakten hat die deutsche Linguistik nachhaltig geprägt und das Grundkonzept wurde über die Jahre hinweg mit wechselnden Schwerpunkten ergänzt und überarbeitet. So führte zum Beispiel der Pictorial Turn dazu, die Sprechakttheorie in verschiedenen, auch multimodalen Kontexten nutzbar zu machen. Es ist $\mathrm{u}$. a. von „Bildhandeln, Bildhandlung, Kommunikationsakt (mit Bild), Malakt, Bildakt, Bildzeigakt, visueller Sprechakt oder Blickakt“ (Opletalová 2016: 30) die Rede, wenn die Linguistik versucht, die Kommunikation mit Bildern anhand von Searles Theorie zu beschreiben. Diese „Nutzbarmachung linguistischer Konzepte, Modelle und Methoden für die Beforschung des in vorwiegend massenmediale Texte integrierten Bildes“ (Klemm/Stöckl 2011: 9) ist die Aufgabe der sog. Bildlinguistik, welche bereits ein fundiertes und sinnvolles Instrumentarium nutzt, um piktorale Artefakte aller Art zu beschreiben. Zu diesen Artefakten gehören seit wenigen Jahren auch die sogenannten Internet-Memes, die für ,,viele Menschen längst ein fester Bestandteil alltäglicher Kommunikationsroutinen [sind]“ (Johann/Bülow 2018: 1). Sie sind sprachenübergreifend ein relevantes Kommunikationsmittel (cf. z. B. Bülow/Johann 2019; Scheiber 2019; 
Weidacher 2019; Merten/Bülow 2019 u. a. m.) und es existieren bereits erste systematische Zugänge, um sie zu beschreiben.

Als Sprache-Bild-Konglomerate sind sie primärer Untersuchungsgegenstad der Bildlinguistik, können aber auch mittels der Sprechakttheorie analysiert werden. Diese ermöglicht einen systematischen Zugang zur Verwendung der vergleichsweise neuen Kommunikate. Mit ihrer Hilfe konnten bereits in der Vergangenheit relativ problemlos multimodale Kontexte erschlossen werden (cf. z. B. Schmitz 2007). Bezogen auf natürliche Sprachen kann „[d]ie Sprechakttheorie [...] zu der Frage nach den sprachlichen Realisierungsmöglichkeiten einzelner Sprechhandlungsmuster einen erheblichen Beitrag liefern“ (Staffeldt 2008: 120). Im Sinne der „InternetLinguistik“ (Marx/Weidacher 2014) soll versucht werden, ,die Theorien die sich bereits zur Beschreibung von Äußerungsbedeutungen und sprachlichen Handlungen offline bewährt haben, auf die Online-Kommunikation [zu überragen]“" (ibd.: 173).

Im Folgenden wird hierzu zunächst der Begriff des Memes bestimmt und erläutert, um daran anschließend konkrete Memes in der Online-Kommunikation als Sprechakte zu analysieren. Der Beitrag schließt mit dem Fazit, dass Memes zwar durchaus als Sprechakte verwendet werden können, es aber Einschränkungen zum traditionellen Verständnis der Sprechakttheorie geben muss.

\section{Der Ausdruck Meme}

Der Fachausdruck Meme oder Mem wurde in der englisch- und deutschsprachigen Forschung vor allem durch Richard Dawkins bekannt (1978). Es stehen sich die beiden englischen Ausdrücke meme [mi:m] (Sg.) - memes [mi:mz] (Pl.) und die in der deutschen Forschung verwendeten Lexeme Mem ['me:m] (Sg.) - Meme ['me:mə] (Pl.) gegenüber. Für die Linguistik ist es wichtig, sich von dem deutschen Wort Mem zu lösen, um zu weit führende Analogien zur MemTheorie von Dawkins (1978) zu vermeiden.

Dieser hatte das Mem ursprünglich zum Gen in Kontrast gesetzt, um eine quasi kulturelle Genetik zu konstruieren. Meme waren für ihn „Melodien, Gedanken, Schlagworte, Kleidermoden, die Kunst, Töpfe zu machen oder Bögen zu bauen“ (Dawkins 1978: 227) und das Wort Mem entstand aus der Verkürzung der konstruierten Ableitung Mimem (von Imitation) in Anlehnung an das Wort Gen (cf. Dawkins 1978: 226). Die „Fruchtbarkeit“" (Dawkins 1978: 229) ist für ihn die ausschlaggebende Eigenschaft, also die Möglichkeit zur Evolution, die auch Shifman (2014) als hochgradig relevant für Memes ansieht. Dieser Grundgedanke steckt auch noch im Konzept Internet-Meme, welches sich allerdings so weit von Dawkins emanzipiert hat, dass sich viele AutorInnen in der Pflicht sehen, auf grundlegende Unterschiede hinzuweisen (cf. exemplarisch Ciorli 2017: 1): „What is important here is that the Internet meme has emancipated itself as an independent creativity-based species, now omnipresent in online reality" (Dynel 2016: 661). Während es in englischsprachigen Veröffentlichungen weiterhin nötig ist, derartige Distanzierungen vorzunehmen, kann im deutschsprachigen Raum darauf verzichtet werden, da die Abgrenzung zu Dawkins bereits mit der Wahl des Lexems geschieht.

\section{$3 \quad$ Was ist ein Meme?}

Laut Davison kann ein Meme ,almost anything“ sein (2012: 126) und auch semiotisch sind Memes schwer bestimmbar: 
Ein Mem [sic] kann beispielsweise ein Bild oder eine [sic] Video sein, eine Phrase, ein längerer Text, ein Dialogschema, eine Textstruktur oder ein immer wieder eingesetzter Rechtschreibfehler sein [sic]; im Grunde kann alles, was imitiert und variiert werden kann, zum Mem [sic] werden.

(Löber 2011: 60)

Auch andere Autoren sehen eine gewisse Unbestimmtheit in der Gestalt (cf. Ciorli 2017: 1), die der Erforschung nicht zuträglich ist. Verkürzt gesagt, ist ein Meme ein kleines, meist bildliches Artefakt, welches zur Kommunikation im Internet genutzt wird:

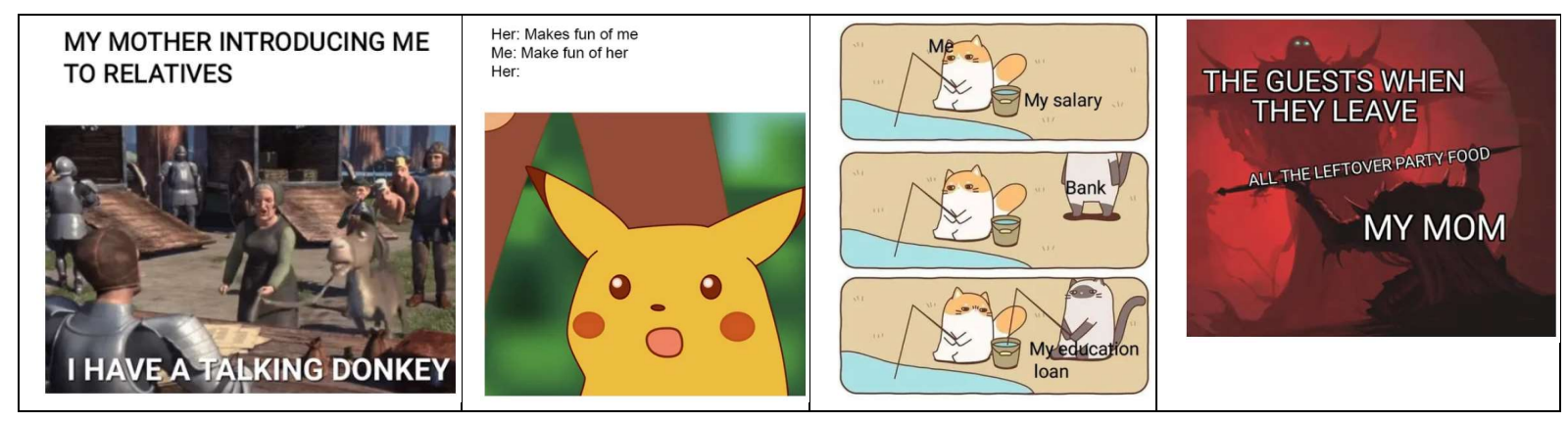

Abbildung 1: Beispiel-Memes

Derartige Memes werden in Online-Gruppen geteilt, wo sie dann von anderen Upvotes oder Downvotes erhalten, je nachdem wie erfolgreich das Meme ist. Während derartige Präsentationsplattformen eher zur Meme-Verbreitung dienen, können sie auch in direkter Kommunikation genutzt werden, dazu aber später mehr.

Diese Unbestimmtheit in der Gestalt, kombiniert mit multimodalen Aspekten und der Viralität von Memes macht es nötig, dass Memes aus verschiedenen Blickwinkeln betrachtet werden, bevor man zu einer Definition kommen kann. So muss die Semiotik des Memes betrachtet werden aber auch das Meme als Text und seine Viralität. Um die Analyse aus linguistischer Sicht überhaupt möglich zu machen, seien zunächst die sogenannten Image Macros betrachtet.

\subsection{Image Macros}

Gut beschrieben ist mittlerweile das sogenannte „Image Macro“ (cf. u. a. Merten/Bülow 2019: 199), welches auch in diesem Beitrag der Ausgangspunkt sein soll. Dies soll nicht bedeuten, dass Image Macros die einzigen untersuchenswerten Memes sind, der Grund liegt vielmehr auf der Zugänglichkeit, da bewegte Bilder sehr viel schwieriger darzustellen sind. Image Macros basieren auf einem Bild, welches einen großen Einfluss auf die Nachricht hat und prototypischerweise auf 2 Textzeilen: 

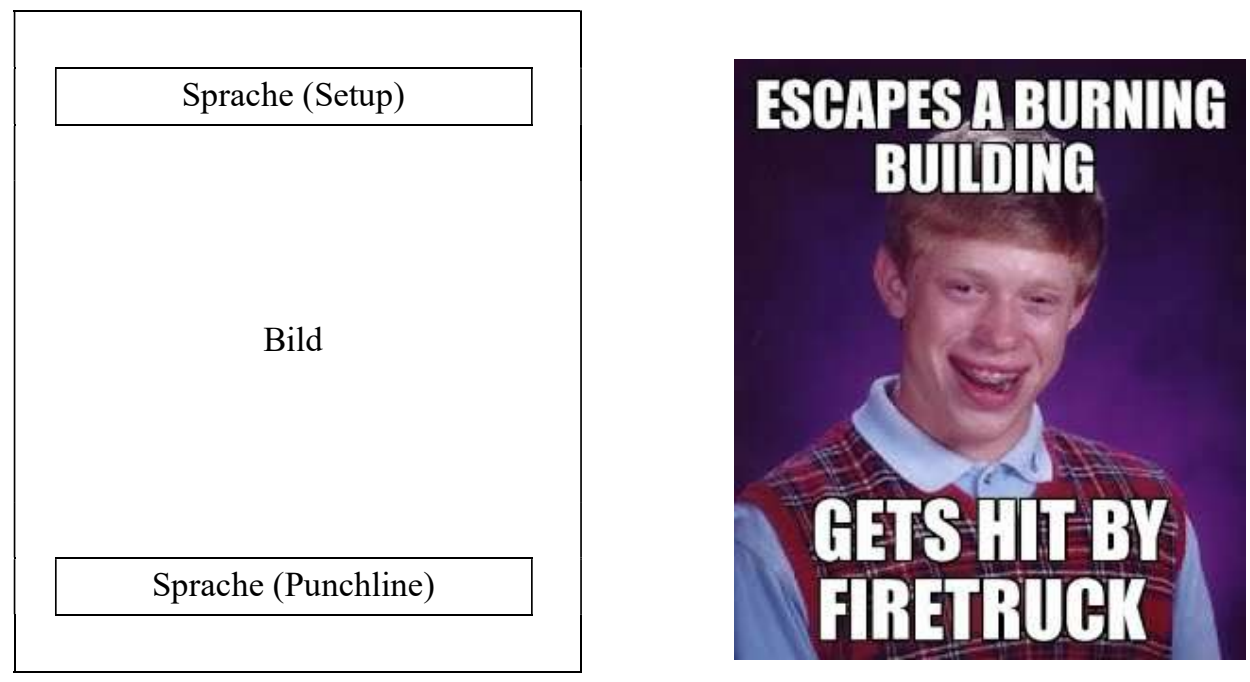

Abbildung 2: Prototypisches Image Macro (cf. u. a. Merten/Bülow 2019: 199)

Das zu Grunde liegende Bild kann der Popkultur, dem aktuellen politischen Geschehen, aber, wie in diesem Fall, durchaus einer zuvor unbekannten Quelle entspringen. Über dem Bild befindet sich das sprachliche Setup, welches einen Frame eröffnet. In diesem Beispiel, dass jemand aus einem brennenden Gebäude entkommen ist. Die Punchline ist, dass die Person vom Feuerwehrwagen überfahren wird. Dieses Pech bzw. Unglück erläutert auch die Namensgebung des Bad Luck Brian-Memes, welches immer dann verwendet wird, wenn Situationen dargestellt werden, in denen der Protagonist großes Pech hat.

Das Bild kann bei derartigen Konstruktionen bedeutungsunterstützend sein, muss es aber nicht. Durch die Verwendung mit immer wieder ähnlichen Setup-/Punchline-Kombinationen konventionalisiert sich die Verwendung des Memes und der Junge auf dem Bild steht irgendwann auch ohne die sprachlichen Ergänzungen für unglückliche Situationen. Der Bild-Sprache-Einheit wird durch die User eine relativ hohe pragmatische Strenge zugewiesen, das Image Macro kann also nur in ganz bestimmten Kontexten verwendet werden (cf. Osterroth 2015). Die Form-Bedeutungs-Konstruktion des Memes befindet sich hierbei allerdings im Fluss, wobei die Nutzer vor allem beim Text einen hohen „Gestaltungsspielraum“ (Merten/Bülow 2019: 199) haben. Das Bild zu verändern ist erst möglich, wenn das Meme schon sehr weit in seiner Konventionalisierung vorangeschritten ist (cf. Osterroth 2015), was uns zur semiotischen Entwicklung der Memes führt.

\subsubsection{Meme-Semiose}

Internet-Memes sind virale Phänomene, welche nicht von Einzelnen erschaffen werden können. Die Meme-Semiose (cf. Herwig 2010: 10; Osterroth 2015: 5; Merten/Bülow 2019: 199) ist ein wichtiger Bestandteil des Phänomens und sprechakttheoretisch interessant. Einzelpersonen können nur meme-fähige Artefakte erschaffen, das mögliche Meme ist ein virales Phänomen und der Schwarm, bzw. die NutzerInnen bestimmen unbewusst über Phänomene der dritten Art, welche Artefakte zu Memes werden: „memes rely on networks of users to spread them to others in a social media ecosystem“ (Leavitt 2014: 145). Ohne diese Netzwerke gäbe es keine Memes. Dieser Vorgang ist relativ komplex und keinesfalls vollständig erforscht, wird aber im Folgenden zumindest theoretisch beschrieben. 


\subsubsection{Semiotische Vertiefung des Memes}

Stufe 1 - Meme-fähiges Artefakt: In dieser Stufe erschafft eine einzelne UserIn oder eine Gruppe ein potenzielles Meme bzw. ein Meme-fähiges Artefakt. Das geschieht täglich tausendfach. Die technischen Hilfsmittel sind frei im Netz verfügbar und intuitiv zu bedienen. In den meisten Fällen wird es sich um ein Image Macro handeln.

Stufe 2 - Teilen und Semiotisieren des Artefakts: Durch wiederholtes Teilen des Memes lernen es immer mehr NutzerInnen kennen und verwenden es selbst. In dieser Phase der Memes-Genese werden meist nur die sprachlichen Elemente angepasst und es werden neue Setups und Punchlines genutzt.

Stufe 3 - Voll konventionalisiertes Meme: Das Meme ist der „Memesphere“ (Miltner 2011: 13) bekannt und wird von vielen NutzerInnen zur Kommunikation herangezogen. Es ist in den Meme-Generatoren verankert und für alle BenutzerInnen verfügbar.

Stufe 4 - Variation des Bildanteils; Streichung des sprachlichen Anteils: Wenn ein Meme vollständig konventionalisiert ist, kann auch das Bild verändert werden. Hier wird z. B. der Kopf der Ursprungsfigur ausgetauscht:

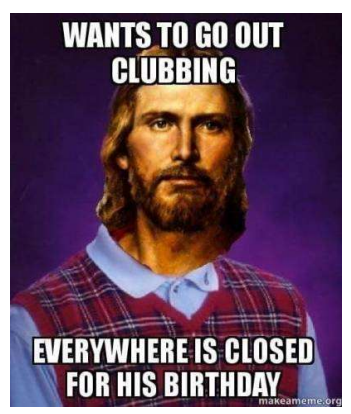

Abbildung 3: Variation des Image Macros

Auch ist es möglich, dass Memes ohne sprachliche Anteile verwendet werden. Wenn z. B. jemand eine Situation erlebt, in der er oder sie großes Pech hat, wäre es angebracht, einfach das Bild des Bad Luck Brian-Memes ohne Text zu senden. Die Kommunikation würde glücken (siehe auch 3.3: Meme-Kommunikation).

\subsubsection{Semiotische Verbreiterung des Memes}

Die Vertiefung des Memes bedeutet, dass sich das Meme zwar konventionalisiert, aber auch obskure Vertreter entstehen. So ist es denkbar, dass ein Meme derart konventionalisiert ist, dass Iterationen erschaffen werden, die für Unwissende überhaupt nicht mehr als Meme erkennbar wären. Gerade in der Diskussion um Artikel 13 und der damit zusammenhängenden CopyrightProblematik, die Memes innewohnt, wurden Memes komplett ohne Bildanteil genutzt. Neben dieser semiotischen Vertiefung muss aber auch die semiotische Verbreiterung des Memes beachtet werden.

Das ursprünglich verwendete Bild und der dazugehörige sprachliche Anteil kann durch die Memewerdung unter Umständen vollständig neu transkribierbar werden (cf. Osterroth 2015: 33). Der übliche Verlauf eines Memes ist, als Insiderwitz zu starten und nur in einer vergleichsweise kleinen Gruppe verstanden $\mathrm{zu}$ werden. Durch die virale Weiterverwendung und 
Alterierung der ursprünglichen Meme-Adaption wird es in weiteren sozialen Medien verwendet, die eine höhere Reichweite haben:

a meme typically starts off as an inside joke and progresses to a wider community, where it becomes humerous in a more generalized sense, and then is rejected by the original host community, who go on to define themselves via other means.

(Zappavigna 2012: 126)

Dieser Prozess geht so weit, dass das Ursprungsmeme mit den rezenteren Iterationen sehr wenig zu tun hat und man viel über die Memesphere wissen muss, um den Text überhaupt noch entschlüsseln zu können: „Before a meme reaches it's semiotic expiry date, users will add increasingly obscure intertextual references to the template slots in displays of template skills" (Zappavigna 2012: 26).

Um das Meme nun linguistisch analysierbar zu machen, z. B. in Form eines Sprechaktes, muss zunächst bestimmt werden, wie es linguistisch zu erfassen ist. Hier kommt der Textbegriff ins Spiel.

\subsection{Das Meme als Text}

Es ist nicht leicht zu beantworten, „was einen Text zu einem Text macht“ (Marx/Weidacher 2014: 178) und die Multimodalität und Neuartigkeit des Memes macht diese Frage nicht einfacher. In der englischsprachigen Forschung wird bereits ganz selbstverständlich von Texten gesprochen, wenn es um Memes geht: „,T]he majority of memes are part of a complex, interconnected, and esoterically self-referential body of texts referred to as ,the memesphere“" (Miltner 2011: 13). Die deutsche Linguistik diskutiert die Frage, was genau ein Text ist, komplexer, was dazu führt dass der Textbegriff durch „Heterogenität“ und „Widersprüchlichkeit“ geprägt ist (Klemm 2002: 17). Die Erfassung neuartiger Textsorten ist dabei ein besonderes Problem, da ,[d]ie traditionelle Textlinguistik [...]ihre methodischen und theoretischen Grundlagen anhand von Texten in ,alten Medien“ [entwickelt hat]“" (Marx/Weidacher 2014: 177).

Im Folgenden soll ,Text‘ im Sinne von Sandig (2000a) als prototypisches Konzept aufgefasst werden, also mit der Annahme, „dass es typischere und weniger typische Texte gibt“ (Marx/Weidacher 2014: 178). Prototypische Texte sind demnach „rein sprachliche Texte“ (Sandig 2000b: 3) während Randvertreter Fahrscheine, Kassenzettel oder eben Memes sein können. Um dies zu zeigen, seien die wichtigsten Textualitätskriterien (nach Marx/Weidacher 2014 und Sandig 2000b) überprüft, nämlich Kohäsion, Kohärenz, Musterhaftigkeit, Multimodalität und Textfunktion.

\subsubsection{Kohäsion und Kohärenz}

Es ist durchaus möglich sowohl Kohäsion als auch Kohärenz in Sprache-Bild-Texten zu zeigen. So weisen z. B. „die meisten Merkel-Meme-Adaptionen“ eine „hohe Bild-Sprache-Kohärenz [auf]“ (Krieg-Holz/Bülow 2019: 107). In diesem Fall werden Personalpronomen genutzt, um das bildlich inszenierte Gespräch mit dem sprachlichen Anteil zu verbinden. Kohäsion ist in Sprache-Bild-Texten schwieriger nachzuweisen, was Stöckl gezeigt hat (Stöckl 2004: 96-98). Hier kann man sich zwar darüber streiten, ob Kohäsion, die ja auf der Textoberfläche stattfindet, bei Bildern überhaupt vorhanden sein kann, doch dies ist für die Argumentation, dass Memes Texte sind, gar nicht nötig. Sandig argumentiert nämlich, dass „Kohäsion, d. h. der sprachlich 
hergestellte Zusammenhang zwischen den Textäußerungen, [...] prototypisch, aber nicht obligatorisch [sei]“" (Sandig 2000b: 3).

\subsubsection{Musterhaftigkeit}

Die Musterhaftigkeit von Memes befindet sich in einem Spannungsverhältnis zur Abweichung (cf. Krieg-Holz/Bülow 2019: 105). Wie weiter oben bereits beschrieben, folgen Memes einem sehr regelhaften Aufbau, können jedoch auch von den NutzerInnen variiert werden. Diese Variation ist aber nur möglich, da Memes so musterhaft sind. So kann es den Prototypen des BadLuck-Brian-Memes geben oder Randvertreter, die nur noch über Familienähnlichkeiten im Sinne Wittgensteins mit diesem Prototypen verbunden sind. Das vereinzelte Aufbrechen der Musterhaftigkeit mit obskuren Vertretern ist gleichzeitig ein Beweis dafür, dass die typischen Memes ganz bestimmten Mustern folgen. Diese Musterhaftigkeit drückt sich in einer gewissen syntaktischen Strenge, den Aufbau des sprachlichen Anteils betreffend, und einer möglichen pragmatischen Strenge, also die Verwendung betreffend, aus (cf. Osterroth 2015: 34-35).

\subsubsection{Multimodalität}

Marx/Weidacher stellen fest, dass „Kombinationen aus sprachlichen, bildlichen und zum Teil auch anderen semiotischen Elementen [so oft zu finden sind], dass diese Form der Multimodalität als typisch für Texte im Internet angesehen werden kann“ (Marx/Weidacher 2014: 188). Die semiotische Perspektive kann in diesem Zusammenhang eine Hilfe sein: „Texte sind demnach alle zeichenhaften Äußerungen, egal welcher medialen Provenienz" (Krah 2013: 31). Linguistisch gesehen kann man dem zustimmen, auch wenn es schwierig ist, Texte mit bildlichen Anteilen begrifflich zu fassen. Wichtige Beiträge dazu haben Ausdrücke geleistet, wie:

- „Texturen“ (Maiwald 2004:105)

- „Gesamttext“ (Doelker 2006: 61)

- „Sehfläche“ (Schmitz 2011: 23)

- „Kommunikat“ (Diekmannshenke 2011: 180)

- „Sprache-Bild-Text“ (Stöck1 2011: 111f.)

Mit dieser Diskussion geht der Ausdruck Bildlinguistik einher, der auf einen Sammelband mit gleichem Namen zurückgeht (cf. Opletalová 2016: 31; Klemm/Stöckl 2011). Dessen Ziel ist es aber keinesfalls ist, eine neue Subdisziplin zu schaffen. Die Bildlinguistik ist kein begrifflich etablierter, linguistischer Bereich, spielt aber innerhalb der Sprachwissenschaft seit einigen Jahren eine große Rolle:

„Bildlinguistik“ - dieser griffige Ausdruck drückt einen Widerspruch in sich aus. Sprache und Bild sind zwei medial, semiotisch, methodisch und technisch verschiedene Ausdrucksformen. Wer spricht, zeigt nicht; wer schreibt, malt nicht. Jedes Kind kennt den Unterschied und weiß ihn gut einzusetzen.

(Schmitz 2011: 2)

Auch wenn Schmitz der Bildlinguistik grundsätzlich kritisch gegenübersteht, ist er der Meinung, dass „,multimodale Kommunikation, also Verständigung über parallele Kanäle und mit mehreren Sinnen, eigentlich der Standardfall [sei]“ (Schmitz 2011:2). Mit den Forschungsinstrumenten der Linguistik ist es möglich, „Wissen und die konstitutiven Regeln explizit zu 
machen, das Musterhafte hinter diesen Zeichenverwendungen $\mathrm{zu}$ erkennen und für verschiedenste Zielgruppen in Wissenschaft und Praxis fruchtbar zu machen" (Klemm/Stöckl 2011: 10).

Bei Memes „konstituiert das Zusammenspiel von bildlichen und sprachlichen Inhaltsaspekten Bedeutung. Es geht daher immer auch um multimodale Metaphern, um die konzeptuelle Integration modedifferenter Bedeutungsinhalte“ (Merten/Bülow 2019: 202). Memes sind also multimodale Texte, die durch Multimodalität überhaupt erst möglich sind.

\subsubsection{Textfunktion}

Sandig stellt fest, dass das „,wichtigste, zentrale Merkmal von Text [...] die Textfunktion [sei]“ (Sandig 2000b: 3), weshalb diese bei Memes auch fokussiert werden soll. Während die oben genannten Eigenschaften in vielen Fällen zutreffen, so haben Memes auf jeden Fall immer eine Funktion, die weiter unten genauer ausgeführt werden soll. Exemplarische Funktionen können das „Beleidigen, Informieren, Belustigen, Zurschaustellung eigener Qualitäten und vieles mehr [sein]" (Osterroth 2015: 33). Wie weiter unten gezeigt werden wird, geht es in der digitalen Kommunikation oft um die Generierung von Aufmerksamkeit, was mit Memes besonders gut gelingt.

\subsubsection{Unterschied zu traditionellen Texten: Variation und Viralität}

Durch neu entstehende Texte wie Memes, aber auch Hypertexte, „,ist [die Textlinguistik] gezwungen, ihre Textdefinition zu überdenken und gegebenenfalls zu adaptieren" (Marx/Weidacher 2014: 177). Neben der Erfüllung der Textualitätskriterien gibt es nämlich Eigenschaften von Memes, die sie klar von prototypischen Texten abheben. Der große Unterschied zum traditionellen Text ist die kollektive und hyperbolisierte Semiose (cf. Herwig 2010: 10). Wie weiter oben beschrieben (siehe 3.1.1), wird ein Meme nicht einfach von Einzelpersonen erschaffen. Vielmehr ist es nötig, dass es sich verbreitet und durch wiederholten Gebrauch konventionalisiert wird (s. o.). Durch das Verbreiten über die sozialen Medien verfestigt es sich in seiner Bedeutung und Funktion. In diesem Zusammenhang handelt sich mitunter um eine eigene Textsorte, oder, je nach Auslegung, um eine Praktik (cf. Krieg-Holz/Bülow 2019). Diese Variation und Viralität macht das Meme als Text einzigartig und rückt es weiter vom Prototypen eines Textes weg.

\subsubsection{Arbeitsdefinition}

Um diesen komplexen, multimodalen und selbstreferentiellen Texten gerecht zu werden, ist es nötig, dass zwei Aspekte des Memes, die bisher erläutert wurden, in die Definition einfließen:

- Sprache und Bild stehen einander ergänzend gegenüber. Dies wird am besten durch Stöckls mittlerweile etablierten Ausdruck „Sprache-Bild-Texte“ erfasst (Stöckl 2004).

- Die Semiose, die eine Bedeutungsentfaltung durch die Nutzer darstellt, ist ein Alleinstellungsmerkmal und führt dazu, dass das Meme nicht als typischer Text wahrgenommen wird. 
Diese beiden Aspekte führen zu folgender Definition:

Internet-Meme sind Sprache-Bild-Texte, deren Bedeutungsentfaltung durch kollektive (oft hyperbolisierte) Semiose stattfindet.

(Osterroth 2015: 33)

\subsubsection{Folgerungen für die Analyse}

Memes als Sprache-Bild-Texte können auch mit Hausendorfs „Kleinen Texten“ (Hausendorf 2009: 6) verglichen werden, da alle von ihm aufgestellten Merkmale zutreffen. Memes sind...

- „kleinräumig-überschaubar“ (ibd.), da sie wenig sprachliche Zeichen nutzen.

- nicht komplex und bestehen ,aus einem Wort, einem Satz oder einem Spruch“ (ibd.)

- auf einen bestimmten „Zweck bezogen“(ibd.).

- „schablonenhaft und stereotyp“ (ibd.).

- sprachlich „unaufwändig“ (ibd.).

Natürlich haben Hausendorfs kleine Texte andere Funktionen und werden eher nicht in der digitalen, sondern in der analogen Kommunikation genutzt, aber es ist ein weiteres Indiz dafür, dass Memes mehr mit bereits erforschten Texten gemeinsam haben, als man vielleicht vermuten mag. Abschließend ist zu sagen, das Memes Randvertreter von Texten im Sinne Sandigs sind, die auch dementsprechend analysiert werden können, was weiter unten mithilfe der Sprechakttheorie geschehen wird. Im Folgenden soll es nun aber zunächst um die Kommunikation mit Memes im Vergleich mit analoger Kommunikation gehen.

\subsection{Meme-Kommunikation}

Internet-Memes werden von verschiedenen NutzerInnen einer Plattform der sozialen Medien geteilt. Dies kann Twitter oder Facebook sein, aber auch Seiten wie 9GAG oder Imageboards auf 4chan. Die Funktionen der Memes sind in den meisten Fällen expressiv/emotiv oder appellativ. Das expressive Moment spielt allerdings immer eine Rolle (cf. Weidacher 2019: 178). Insbesondere politische Memes können auch eine Persuasionsabsicht transportieren (cf. Weidacher 2019: 178).

\subsubsection{Unpolitische Memes}

Der humoristische Charakter der meisten unpolitischen Memes legt nahe, dass die Informationsvermittlung hier nicht im Vordergrund steht. Vielmehr sticht ihr expressiver Charakter hervor. Ein viel wichtigerer Aspekt als die Informationsvermittlung ist das Schaffen sozialer Identifikation, z. B. durch „Insiderwitze“ (Zappavigna 2012: 126): „Internet memes are deployed for social bonding rather than sharing information" (ibd.: 101). Hier werden die Memes oft vordergründig zum reinen Spaß generiert, um sich gegenseitig zu belustigen: ,, commonly cited reason for producing a meme is for the , lulz', an act associated with humour that solidifies an ingroup [...] by ridiculing others“ (ibd.). In diesem Zusammenhang sind die Meme-User durchaus als Community of Practice (cf. Wenger 2000) zu verstehen, deren gemeinsame Aktivität ausschließlich das Kommunizieren mittels Memes ist. So werden ,gemeinsame kommunikative Strategien und Vorstellungen von angemessenem sprachlichem Handeln in der Interaktion entwickelt" (Merten/Bülow 2019: 199). Informationsvermittlung ist in solchen Konstellationen nicht das Hauptziel, und bereits Austin stellte in Frage, ob die konstatierende Äußerung 
oder, in seinen Worten, „such an animal“ (Austin 1962: 109) überhaupt existiere. Das Informieren ist ein Sprechakt und in diesem Sinne keiner, auf den er einen besonderen Fokus gelegt hat.

\subsubsection{Politische Memes}

Politische Memes von unpolitischen abzugrenzen ist nicht einfach und „[b]islang liegt noch keine wirklich überzeugende Klassifikation politischer Internet-Memes vor" (Johann/Bülow 2019, S: 22). Es ist nur klar, dass sich nicht alle Genres ,gleichermaßen für die Verwendung in politischen Kontexten verwenden lassen“ (Johann/Bülow 2019: 22). Da es in diesem Beitrag vor allem um die Benutzung auf Twitter geht, kann aber zumindest ein Definitionsversuch unternommen werden, der die folgenden Analysen stützt. Ein Meme sei genau dann politisch, wenn es in einem politischen Kontext, also z. B. als Antwort auf einen politischen Tweet des Präsidenten, genutzt wird oder wenn es politische Akteure oder Zitate abbildet. Da alle hier untersuchten Memes in einem politischen Diskurs genutzt werden, können sie als politische Memes klassifiziert werden. In derartigen Kontexten werden die Sprechakte oft zur „Orientierung des Diskurses und zur Persuasion anderer Kursteilnehmern bzw. zur Konfirmation deren ideologischer Einstellungen [genutzt]“ (Weidacher 2019: 190). Wegen der Schwierigkeit, politische Memes trennscharf von unpolitischen abzugrenzen, bietet es sich an, Memes in Antwortthreads auf Trump zu untersuchen. Da die Äußerungen des Präsidenten per se als politisch anzusehen sind, sind die Antworten auf ebendiese Äußerungen auch politisch und so umgeht man das Problem der Bestimmung.

\section{$4 \quad$ Analyse von Memes in Antwortthreads auf Trump}

\subsection{Twitter als Datenquelle}

Twitter ist eine Plattform, die es den NutzerInnen leicht macht, an politischen und kulturellen Diskussionen teilzunehmen: „Wesentliches Merkmal dieser digitalen Partizipationskultur sind die relativ niedrigen Barrieren zur Teilnahme an verschiedentlichen Online-Kommunikationspraxen“(Merten/Bülow 2019: 195). Durch diese ,niederschwellige Möglichkeit des Meinungsausdrucks“ (Merten/Bülow 2019: 197) sind sogenannte „Graswurzelbewegungen“ (Merten/Bülow 2019: 198) möglich und die BürgerInnen können politische Initiativen sehr einfach ohne großen Aufwand starten. Memes sind ein wichtiger Bestandteil, da auch die technischen Hürden gesunken sind, derartige Texte selbst zu produzieren. „Meme-Generatoren ermöglichen das Vorgeben einer Vielzahl von Templates, die mit Sprache versehen werden können, ein (größtenteils) problemloses Erstellen sogenannter Image Macros“ (Merten/Bülow 2019: 196). Wichtig anzumerken ist dabei, dass Memes damit nicht erschaffen werden, es werden lediglich bereits bekannte Memes genutzt. Geteilte Memes verbreiten sich auf Twitter besonders schnell, da der Algorithmus das Einzugsgebiet der möglichen Rezipienten immer weiter vergrößert:

More recently, many memes spread from a smaller network of users to a broader set of viewers with the aid of Twitter's technical architecture. [...] As memes move between individuals, the cultural salience of the meme increases: it becomes more meaningful for more people.

(Leavitt 2014: 139)

Auf Twitter werden die Beiträge populär, die von vielen Personen geliked und geteilt werden. Die Zustimmung der breiten Masse ist eine digitale Währung: Im Sinne der von Keller 
formulierten Hypermaxime kommunikativen Handelns sind die UserInnen bestrebt, so zu reden, dass sie „sozial erfolgreich [sind]“ (Keller 2003: 142). Diese Wahrnehmungslogik macht Twitter auch für die pragmalinguistische Meme-Forschung interessant. Schließlich geht es bei Twitter auch um den Zusammenhang zwischen sozialem Erfolg und sprachlicher Struktur. Damit ist die Beachtung Kellers weiterer Maximen in diesem Kontext höchst relevanter:

- „Rede so, dass Du beachtet wirst.“ (Keller 2003: 139)

- „Rede amüsant, witzig“ (ibd.)

Likes sind die „neue Währung sozialer Anerkennung“(Vorderer 2015: 265). Und „Rede“(Keller 2003: 139) meint in diesem Zusammenhang Sprechakte, die man mit der Welt teilt. Der soziale Erfolg der Anerkennung in den sozialen Medien scheint sich mit Memes sehr gut generieren zu lassen, ,vor allem [weil] emotional aufgeladene und humorvolle Inhalte sowie intertextuelle Bezüge die Weiterleitung begünstigen“" (Johann/Bülow 2018: 6). Neben diesen eher technischen Aspekten muss man Twitter aber auch inhaltlich einordnen. Auf jeder Plattform, auf der die UserInnen selbst Content generieren, finden sich auch immer problematische Inhalte: „Large amounts of hate speech on exists on platforms that allow for user generated documents, which creates a need to detect and filter it" (Waseem 2016: 138).

Memes können durchaus im Bereich der sogenannten Hate-Speech genutzt werden (cf. z. B. die Memes zur Flüchtlingskrise, analysiert in Weidacher 2019). Die niederschwelligen Partizipationsmöglichkeiten, gepaart mit relativer Anonymität führen, wie man auch weiter unten bei den Beispielen auf Trumps Tweets sieht, durchaus zu aggressiven Sprechakten. SentimentAnalysen haben bereits gezeigt, dass Tweets tendenziell eher negativ als positiv sind (cf. Thelwall 2014: 93) und selbst Amnesty International hat bereits auf die „Toxicity“ von Twitter hingewiesen (cf. Amnesty Research Project 2018). ${ }^{1}$

\subsection{Sprache-Bild-Akte / Multimodale Sprechakte}

Laut Schmitz (2007: 419) hat ,,[m]an [...] lange genug angenommen, das Geschäft von Bildern sei einzig und allein, einen Sachverhalt abzubilden“. Das ist aber nicht der Fall und Bilder können ,in mannigfaltigen Sprachspielen [...] und Bildspielen [verwendet werden]“ (Schmitz 2007: 420). Als Ergänzung seien Sprache-Bild-Texte genannt, die genau diese Aufgabe erfüllen können. Laut Opletalová (2016: 28) wird ,[d]as Konzept des Bildhandelns [...] oft sehr vage beschrieben“" und es werden auch zahlreiche neue Begriffe genutzt, um das Phänomen zu erfassen (s. o.). Die Erforschung von Bildakten im Sinne der Sprechakttheorie hat bereits eine sehr fundierte Forschungstradition, auf die sie zurückblicken kann. Einige wichtige Arbeiten dazu sind z. B.: Sachs-Hombach (2011; 2003), Krämer (2009), Schmitz (2007), Lauschke (2013), Opletalová (2016), Grundlingh (2017). In Bezug auf die extensive Forschung zu Bildakten würden sich im Folgenden die Ausdrücke Meme-Sprechakt, Bildakt oder auch Meme-Akt anbieten. Der Lesbarkeit wegen wird jedoch möglichst Sprechakt genutzt, ohne dabei den multimodalen Charakter der Image Macros auszuschließen. Vielmehr wird vorausgesetzt, dass Sprechakte ohnehin immer multimodal sind. Zudem werden im Folgenden neben den

\footnotetext{
${ }^{1}$ Ergänzend sei der Comedian Ricky Gervais genannt, der folgendes zu Twitter meint: „Twitter is like being able to read every toilet wall in the world“ (Gervais 2013). Auch wenn diese Aussage sicherlich zugespitzt ist, so lässt sich dies anhand der Beispiele von Amnesty International sicherlich nachvollziehen.
} 
unbewegten, klassischen Image Macros auch sogenannte GIFs analysiert, also bewegte Bildfolgen, die sich stets wiederholen und einem sehr ähnlichen Aufbau folgen wie prototypische Memes. Anstelle von zwei sprachlichen Zeilen wird jedoch oft nur eine Punchline genutzt, die darstellt, was die Protagonisten sagen, da GIFs keinen Ton haben. GIFs können als Subklasse von Image Macros betrachtet werden.

\subsection{Sprechakte in einem Antwortthread von Trump}

Memes über Donald Trump sind sehr beliebt und werden deshalb aufgrund ihrer hohen Verbreitung und Sichtbarkeit in den sozialen Medien häufig als Forschungsgegenstand gewählt (cf. Pauliks 2019). Die folgende Analyse befasst sich jedoch nicht mit Memes über Trump, sondern mit Memes, die als Antworten auf Tweets des US-Präsidenten gesendet werden. In den entstehenden Threads auf Twitter gibt es mehrere zehntausend Antworten und für diesen Beitrag wurden nicht alle ausgewertet. Deshalb muss an der Stelle auf eine quantitative Analyse verzichtet werden, stattdessen werden die Memes qualitativ analysiert. Die hier untersuchten Memes entstammen Threads, die als Antworten auf folgende Tweets von Donald Trump entstanden sind:

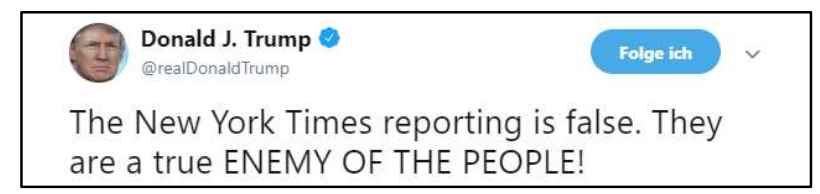

Abbildung 4: Trump vom 20.2.2019

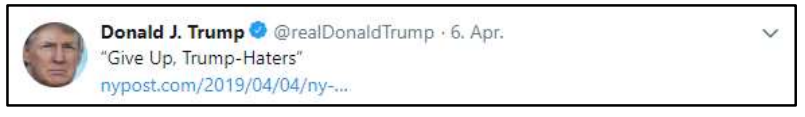

Abbildung 5: Trump vom 06.04.2019

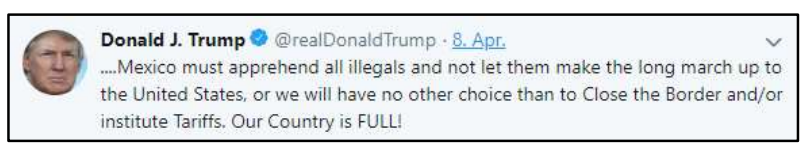

Abbildung 6: Trump vom 08.04.2019

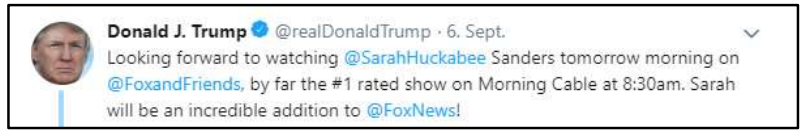

Abbildung 7: Trump vom 06.09.2019

Über dieses Profil twittert Donald Trump weitestgehend nicht kuratiert und interagiert so mit seinen FollowerInnen. Genauso ungefiltert wie die Tweets vom Präsidenten veröffentlicht werden, genauso ungefiltert sind die Antworten. Für diese Untersuchung wurde der Thread betrachtet, aber nur eine kleine Auswahl an Memes herausgesucht, um zu erläutern, inwiefern Memes als Sprechakte in der Klassifikation von Searle (cf. 1965; 1982; 1984) verwendet werden.

\subsubsection{Assertiva}

Assertiva bzw. Repräsentativa sind Sprechakte, die ausdrücken, ,dass die zum Ausdruck gebrachte Proposition wahr ist“" (Searle 1982: 31). Dazu gehört die Möglichkeit, dass die 
„entsprechende Proposition p wahr oder falsch sein kann“ (Hindelang 2010: 45). Häufige assertive Sprechakte sind Behauptungen, Beschreibungen oder Feststellungen.

Sie kommen in Meme-Form nicht häufig vor, da es selten das Ziel ist, eine wahre Proposition auszudrücken, wenn man auf die Tweets anderer antwortet. Stattdessen haben auch Assertiva stets etwas Expressives an sich, da auch immer die Einstellung des Senders mit ausgedrückt wird. Neben der ,psychischen Einstellung [und dem] Glaube[n], dass p wahr ist“ (Hindelang 2010: 45) wird auch eine Meinung zu dem Thema mit transportiert.

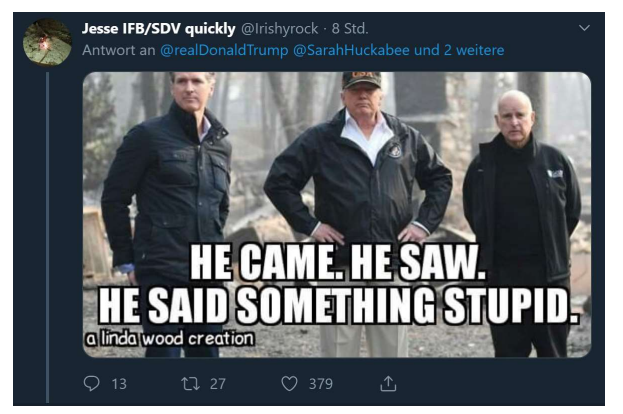

Abbildung 8: Beispiel für ein assertives Meme

Das Meme in Abbildung 8 ist ein assertives, da die geäußerte „Proposition p wahr oder falsch sein kann. [...] Die Worte müssen den Tatsachen entsprechen“"(Hindelang 2010: 45). In diesem Falle ist es eine Feststellung, die natürlich auch eine ,psychisch[e] Einstellung“ (Hindelang 2010: 45) übermittelt und die Annahme stärkt, dass es sich bei Meme-Sprechakten oft auch um expressive Sprechakte handelt. Der assertive Charakter überwiegt jedoch, weshalb es zu dieser Klasse gezählt werden kann.

\subsubsection{Direktiva}

Direktiva sind „Versuche des Sprechers [...], den Hörer dazu zu bekommen, dass er etwas tut“ (Searle 1982: 32). Handlungsaufforderungen in Meme-Form werde auf Twitter selten mit ernst gemeinter Absicht verwendet. Den meisten NutzerInnen ist bewusst, dass ihre Aufforderung auf keiner Konvention fußt. Jeder weiß, dass man z. B. dem Präsidenten der Vereinigten Staaten keinen Ratschlag geben kann, es wird aber ironischerweise dennoch getan. Die meisten direktiven Sprechakte richten sich jedoch nicht an Trump selbst, sondern eher an die Gemeinschaft derer, die sich unter seinen Äußerungen versammeln:

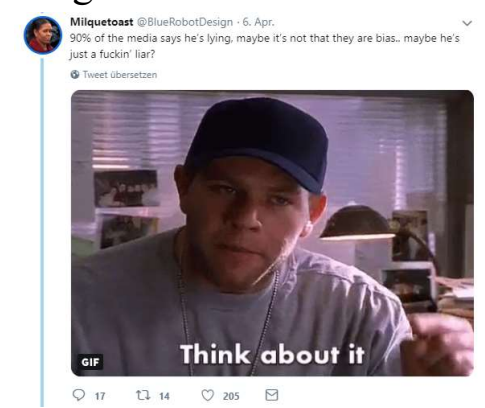

\section{Abbildung 9: Beispiel für ein direktives Meme}

Bei dem Meme in Abbildung 9 handelt es sich um ein bewegtes GIF, also eine kurze, sich wiederholende Animationsfolge, welches prototypisch nur eine Textzeile hat, die meist der Text der gezeigten Filmszene ist. Hier ist ein Screenshot abgebildet, der den Sprechakt 
nachvollziehbar transportiert. „Denk darüber nach“ ist ein direktiver Sprechakt, da „der Sprecher [...] den Hörer zur Ausführung einer zukünftigen Handlung verpflichten [will]“ (Hindelang 2010: 45). Der Sprechakt richtet sich in diesem Fall aber nicht an Trump, sondern an die anderen NutzerInnen im Diskurs, die sich im selben Thread befinden.

\subsubsection{Kommissiva}

Wenn der Sender Kommissiva verwendet, dann verspricht er, dass er „eine künftige Handlung [...] vollzieht" (Searle 1982: 33). In diesem Falle schlägt Trump vor, Fox News zu schauen und ein Follower versichert per Meme, dass er das nicht tun wird:

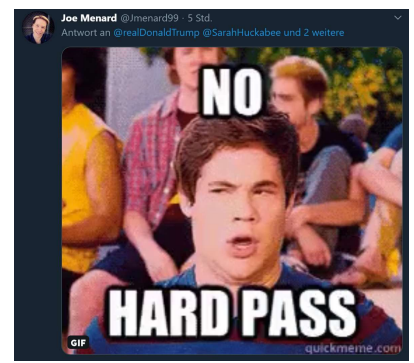

Abbildung 10: Beispiel für ein kommissives Meme

Kommissiva sind relativ selten in den Antwortthreads. Oftmals werden sie scherzhaft und ironisch genutzt, was dazu führt, dass sich die Ersteller eher auf das Nicht-Durchführen einer Handlung festlegen. Oftmals wird dadurch expressiv und eindeutig eine Ablehnung von Trumps ursprünglichem Vorschlag klargemacht.

\subsubsection{Expressiva}

Die Rolle der Expressiva ist es, ,,den in der Aufrichtigkeitsbedingung angegebenen psychischen Zustand zum Ausdruck zu bringen, der auf eine im propositionalen Gehalt aufgeführte Sachlage gerichtet ist“" (Searle 1982: 34). Grundlingh (2017: 150) behauptet daher: „Almost every meme is created with the purpose of expressing something“. Die expressiven Memes sind in dieser Twitter-Diskussion am häufigsten vertreten, da Twitter eine Plattform ist, auf der es in der Hauptsache darum geht, seine Meinung auszudrücken. Generell ist festzuhalten, dass Expressiva den Raum der sozialen Medien bestimmen, während die anderen Sprechakte dagegen seltener vorkommen. Es ,gilt es in der modernen Internetkultur als selbstverständliches Recht und als guter Stil, die eigene Meinung zu äußern“ (Vorderer 2015: 271). Wie Staffeldt (cf. 2008: 84f.) korrekt anmerkt, geht es um „das Schaffen sozialer Beziehungen“ (2008: 85), wenn expressive Sprechakte durchgeführt werden. Im Falle der Memes, die Trump widersprechen, handelt es sich um konstitutive Akte, die die eigene Rolle im Diskurs deutlich machen.

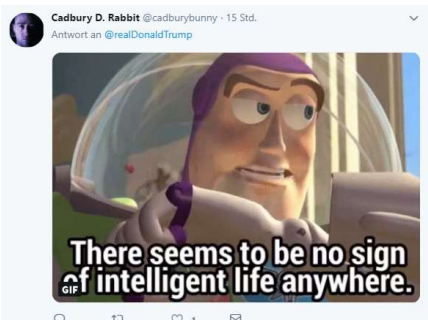

Abbildung 11: Beispiel für ein expressives Meme 
Das Meme in Abbildung 9 gehört der großen Gruppe der Exploitables an. Diese sind sehr unspezifisch und verweisen ,auf einen großen Kreis von möglichen im jeweiligen Kontext aktualisierbaren Bedeutungen“" (Scheiber 2019: 155). Das Bild könnte so in vielen kommunikativen Situationen genutzt werden, die nicht weiter spezifiziert sind. Es ist so unspezifisch, dass es sich nur noch sehr verblasst assertiv auffassen lässt und der expressive Sprechakt stark überwiegt. Es kann genutzt werden, um in einer beliebigen Kommunikation Missfallen über die Inhalte auszudrücken, was es zu einem expressiven Exploitable macht.

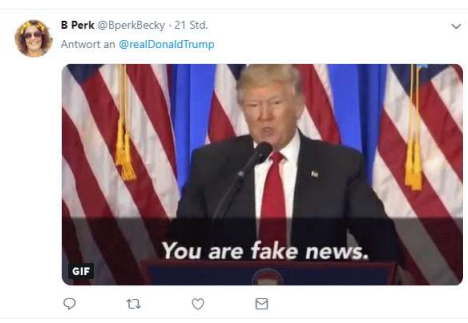

Abbildung 12: Beispiel für ein expressives Meme

Das expressive Meme in Abbildung 10 könnte zunächst assertiv verstanden werden, aber die Nutzung von ,fake news“ ist extrem subjektiv und ein expressiver Akt, da ,[d]er illokutionäre Zweck [...] im Ausdruck einer psychischen Einstellung zu einer Proposition p [besteht]" (Hindelang 2010: 45) und der Wahrheitsgehalt, ob eine Person „,fake news“ ist, sich nicht überprüfen lässt. Dieses Meme hat zudem eine Meta-Ebene, da es nicht nur ein expressiver Sprechakt als Reaktion auf Trump ist, sondern ihn auch in Meme-Form benutzt. Hier wird eine meme-gewordene Aussage des US Präsidenten ironisch gegen ihn gewendet, vor allem, wenn man bedenkt, dass er in seinem Tweet die Medien kritisiert.

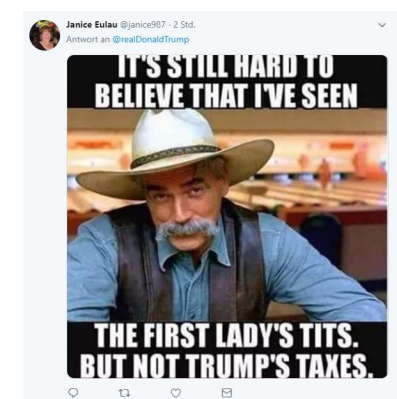

Abbildung 13: Beispiel für ein prototypisches expressives Meme

Nachdem die ersten beiden Beispiele eher untypische Vertreter des Image Macros waren, handelt es sich hier um eine sehr prototypische Variante, bei der ein popkulturelles Bild mit zwei Sprachzeilen ergänzt wurde. Auch dies ist eine Form des Exploitables, da es syntaktisch sehr frei ist. ${ }^{2}$ Es gibt weitere Image Macros, deren Syntax teilweise bestimmt und stark konventionalisiert ist (cf. Osterroth 2015), aber in diesem Fall sind sowohl Setup als auch Punchline syntaktisch nicht festgelegt.

Da das Meme mit „Es ist schwer zu glauben, dass...“ eingeleitet wird, drückt es relativ klar eine psychische Einstellung aus und lässt sich somit den Expressiva zuordnen. Es findet sich aber auch ein direktives Element, da das Meme unter Umständen als Aufforderung verstanden

\footnotetext{
2 Zu den Begriffen syntaktische Strenge und pragmatische Strenge cf. Osterroth (2015).
} 
werden kann, sich mit den genannten Themen, also hier mit den Steuern Trumps, zu beschäftigen. Dieses Abgrenzungsproblem wird im nächsten Abschnitt diskutiert.

\subsection{Bewertung der Expressivität - Funktion des bildlichen Anteils}

Nicht nur werden viele Memes dafür genutzt, expressive Sprechakte durchzuführen, auch scheint es so zu sein, dass die anderen Sprechakte stets eine expressive Komponente haben. Jedes Meme ist neben seiner Funktion als Sprechakt auch eine sogenannte „E-Implikatur“ (Schwarz-Friesel 2009: 227). Hierbei wird zu dem eigentlichen Sprechakt der ,emotiv[e] Beziehungsaspekt" (Schwarz-Friesel 2009: 227) eingebracht, wie es auch bei Ironie der Fall ist. Die Verwendung von Bildern unterstützt dies zusätzlich. Für die SenderInnen der Memes ist „[d]er Ausdruck von Gefühlen [...] visuell leichter möglich als in sprachlicher Form, die den Gefühlsausdruck stärker an bestimmte grammatikalische und semantische Ablaufkonventionen bindet“ (Kappas/Müller 2006: 3). Bilder „lösen emotionale Reaktionen aus“ (Kappas/Müller 2006: 3) und „,[m]it Bildern kann man auch besser emotionalisieren“ (Marx/Weidacher 2014: 190).Im Falle der Memes werden die Emotionen, die man durch das Bild empfindet, auf die SenderInnen übertragen. Wenn man also bspw. ein Bild von einer vorwurfsvoll schauenden Person für sein Meme nutzt, dann wird diese Eigenschaft auf die ErstellerIn projiziert.

Dadurch lassen sich Memes welcher Art auch immer schwer von expressiven Sprechakten abtrennen. Dies ist aber kein neues Problem sprechakttheoretischen Analysen. Wenn man sich z. B. den von Spitzmüller/Warnke analysierten Menschenrechtsdiskurs ansieht, dann kann es sein dass Sprechakte auf den ersten Blick assertiv scheinen, obwohl die „Funktion des Satzes durchaus direktiv [ist]“ (Spitzmüller/Warnke: 148). Am besten lässt sich die Überlappung der Expressivität der Internet-Memes mit dem ,,illokutionären Zweck (illocutionary point)“ (Hindelang 2010: 44) erklären. Die „kommunikativen und praktischen Absichten“ (Hindelang 2010: 44) der NutzerInnen auf Twitter sind stets auch expressiv und selten deckungsgleich mit den traditionellen Absichten von Sprechakten. Dass die Sprechaktklassen, so elaboriert sie auch sein mögen, einander generell überlappen, wird im Fazit erneut aufgegriffen (siehe 5).

\section{$5 \quad$ Memes als Sprechakte?}

Dass Internet-Memes das Potenzial haben, Sprechakte zu sein, ist in der englischsprachigen Forschung schon länger bekannt, wie Grundlingh (2017: 154): „Although all meme types could potentially be interpreted as speech acts, it is true that some types are easier to interpret than others and are also used with more frequency“. Der wichtigste Faktor bei der Nutzung von Memes als Sprechakten ist die Konventionalisierung, die im Folgenden näher erläutert werden soll.

\subsection{Konventionalisierung}

Auch bei rein sprachlichen Sprechakten gelingt die Illokution nur, wenn eine Konvention vorliegt, das bedeutet, dass das gemeinsame, kulturelle Wissen vorhanden sein muss. Nicht umsonst scheitern Sprechakte sehr häufig vor allem in interkulturellen Situationen. Konventionen sind ,immer sozial und gruppenspezifisch“ (Heringer 2014: 33). In der gesprochenen Sprache spielen Kulturen und Subkulturen eine große Rolle, auch verschiedene Nationalitäten haben unterschiedliche Common Grounds, aus denen sie pragmatische Konventionen ziehen (cf. 
Heringer 2014: 166). Common Ground kann definiert werde als ,a body of information that is presumed to be shared by the parties to a discourse" (Stalnaker 2014: 4). Im Falle der Memes ist neben der allgemeinen Popkultur und Kultur ein wichtiger Common Ground die sog. „Memesphere“ (Miltner 2011: 13), die sich im Gegensatz zu kulturellen Quellen aber in einem sehr viel dynamischeren Fluss befindet und einem enorm schnellen Wandel unterliegt. ${ }^{3}$ Je öfter ein Meme verwendet wird, desto besser stehen die Chancen, dass Bestandteile konventionalisiert verwendet werden. Nach einer nicht genau zu bestimmenden Anzahl von Variationen erreicht das Meme einen Saturationspunkt und kann von vielen NutzerInnen für ihre jeweiligen kommunikativen Zwecke genutzt werden. Die Kommunikation mittels eines Memes kann dann glücken, wenn Sender und Empfänger genug Wissen aus der Memesphere teilen.

\subsection{Einschränkungen}

Während rein sprachliche Sprechakte die tägliche Kommunikation ausmachen und, wie Austin bereits sagte, sprachliches Handeln darstellen, so verhält es sich mit den Meme-Sprechakten in vielen Fällen anders.

Die meisten Memes sind als expressive Sprechakte zu klassifizieren oder zumindest als Sprechakte mit einem expressiven Anteil. Wenn man mit Austin sprechen will, dann haben MemeNutzer andere perlokutionäre Wirkungen im Sinne als man von der Illokution traditionellerweise erwarten würde. Den SenderInnen ist klar, dass eine perlokutionäre Wirkung auf z. B. eine Warnung unwahrscheinlich ist. Alle KritikerInnen in dem Thread von Trump wissen, dass der US-Präsident nicht auf die Antworten reagieren wird, zumindest nicht, wie es bei einem traditionellen Face-to-Face-Gespräch der Fall wäre.

Wie bereits in einigen Beiträgen gezeigt wurde, handelt es sich bei den Memes oft um Begleiterscheinungen oder gar Auslöser von „Graswurzelbewegungen“ (Merten/Bülow 2019: 198). Mithilfe von Memes ist es den KommunikationsteilnehmerInnen möglich, kommunikativ an der politischen Welt teilzunehmen, da Memes eine ,niederschwellige Möglichkeit des Meinungsausdrucks [darstellen]“" (Merten/Bülow 2019: 197). Dies ist jedoch eine Eigenschaft jeder Kommunikation in den sozialen Medien.

Das schränkt die pragmatische Funktionalität des Meme-Sprechaktes keinesfalls ein, da es sicher auch viele private Chats gibt, in denen Memes als Sprechakte im traditionellen Sinne genutzt werden. Dies zu erforschen ist jedoch eine große Herausforderung, da es nachvollziehbare, datenschutzrechtliche Bedenken gibt, private Chats mit Bildern zu speichern. Rein sprachliche Korpora sind bereits vorhanden (z. B. MoCoDa2), aber für die Meme-Forschung leider nicht nutzbar.

Die öffentlichen und deswegen gut beschreibbaren Memes, in diesem Falle auf Twitter, sind vor allem als subversive Sprechakte zu verstehen.

\subsection{Memes als subversive Sprechakte}

Memes sind inhärent kritisch. Es ist zwar möglich, mit Memes aufrichtige Sprechakte zu senden, dies ist aber nicht der Standardfall:

\footnotetext{
${ }^{3}$ Die Memesphere selbst fußt zudem noch auf weiteren, popkulturellen Common Grounds, aber diese Vertiefung würde hier zu weit gehen.
} 
Because memes are a unique way of communicating, it is obvious that the functions of memes will not be able to correspond to all the communicative illocutionary acts identified. However, memes can be created for specific and personal purposes. This means that a meme that thanks someone or congratulates someone in a sincere manner is completely possible.

(Grundlingh 2017: 161)

Es ist untypisch, ernstgemeinte Memes in der Kommunikation zu nutzen, es schwingt fast immer Humor und Ironie mit (cf. Osterroth 2019). Dies geht zurück auf die Tradition der Emoticons, mit denen NutzerInnen des frühen Internets scherzhafte Nachrichten markiert haben (cf. Ciorli 2017: 4). Dies ist heute per Meme-Sprechakt möglich, da das Meme als Textsorte bereits Humor und Unernsthaftigkeit inkorporiert.

Neben dieser ironischen Verwendungsweise werden Memes häufig als politischer Kommentar genutzt und Johann/Bülow (2019) stellen sehr berechtigt die These auf, dass Memes in einer Tradition politischer Fotografie stehen. Im Gegensatz jedoch zur klassischen Fotografie, die nur JournalistInnen möglich war, können die NutzerInnen heute auf einfachste Weise politische, subversive Kommentare in die Welt senden. Dass diese Sprechakte durchaus ernstgenommen werden, zeigen Meme-Verbote jüngerer Zeit. In China wurde ein Winnie the Pooh-Meme, welches genutzt wurde, um sich über den Präsidenten Xi lustig zu machen, verboten (cf. Siegel 2018). Auch Russland verbietet staatskritische Memes schon seit längerer Zeit und in Nordkorea sind ironische Inhalte ohnehin verboten (cf. Dewey 2015). Diese Verbote sind ein weiteres Indiz dafür, dass mit den Memes tatsächlich gehandelt wird und macht die Frage „How to do things with Memes?“"(Osterroth 2019) noch aktueller.

\section{$6 \quad$ Fazit}

Es hat sich gezeigt, dass die Sprechakttheorie ein zentrales und geeignetes Analysetool zur Bestimmung der Meme-Kommunikation ist. Die Klassifikation der Memes nach Searle lässt zwar wichtige Eigenschaften, wie die Multimodalität und die Viralität der Memes aus, zeigt aber, dass fast alle Memes expressiv sind. Dass die Searle'schen Klassen sich überlappen ist keine neue Erkenntnis (vgl Hindelang 2010: 43), aber es wurde, zumindest beispielhaft, klar, dass sich Internet-Memes kaum ohne expressives Moment verwenden lassen. Das führt dazu, dass sich Meme-Sprechakte noch weniger sauber klassifizieren lassen als rein sprachliche.

Dieses Problem ist jedoch ein grundsätzliches. In der Sprechakttheorie ist es ,gar nicht möglich, eine eindeutige, ,wasserdichte" Abgrenzung zu schaffen“" (Hindelang 2010: 43). Neben den Klassen von Austin und Searle werden ,[bis heute] Vorschläge zu einer exhaustiven Typologie [...] vorgelegt und diskutiert, ohne dass es einen abschließenden Konsens gäbe“" (Stukenbrook 2013: 220). Neue Klassen sorgen nicht unbedingt dafür, dass die Abgrenzungsproblematik verschwindet, was aber auch nicht zielführend wäre. Da die Sprechakttheorie ohnehin nicht „,empirisch fundiert“ (Stukenbrook 2013: 220) ist, sondern vielmehr auf der „Intuition von Wissenschaftlerinnen und Wissenschaftlern" (Stukenbrook 2013: 220) basiert, trägt die Analyse von Internet-Memes zum Gesamtkomplex Sprechakttheorie bei. Es wird deutlich, dass sich diese Kommunikate in den meisten Fällen mehreren aber fast immer der expressiven Klasse zuordnen lassen.

Insgesamt konnte dieser Beitrag zeigen, dass sich die Verwendungsweise der Meme-Kommunikation mit der Sprechakttheorie erläutern lässt und linguistisch relevant ist. Für die zukünftige 
Forschung wäre es wichtig, die Meme-Kommunikation auch großflächig empirisch zu erheben, um dann konkrete Schlüsse im Sinne der Sprechakttheorie ziehen zu können. Wie weiter oben erwähnt, stehen der Analyse privater Kommunikation der Datenschutz und technische Limitationen im Wege, aber in Bezug auf politische Memes ist es möglich, öffentlich zugängliche Diskurse zu analysieren. Der Erkenntnisgewinn, der sich daraus ergeben könnte, würde eine vertiefende theoretische Beschäftigung mit Memes ermöglichen, die über die hier gezeigten Analysen weit hinaus gehen würde.

\section{Literaturverzeichnis}

Amnesty Research Project (2018): Toxic Twitter. Chapter 1: A Toxic Place for Women. amnesty.org/en/latest/research/2018/03/online-violence-against-women-chapter-1, -2, -3, -4, $-5,-6,-7$ and -8 . [03.03.2020].

Austin, John L. (1962): How to do things with words. The William James Lectures delivered at Harvard University in 1955. Oxford: Clarendon Press.

Bülow, Lars/Johann, Michael (eds.) (2019): Politische Internet-Memes - Theoretische Herausforderungen und empirische Befunde. Berlin: Frank \& Timme. (= Texte und Diskurse 4).

Ciorli, Marco (2017): “One Does Not Simply Send Memes”. Performativity of Internet Memes in Synchronous Mediated Communication. Trient: University of Trento. (= FSS:SOC254 Chapters in Cultural Sociology).

Davison, Patrick (2012): “The Language of Internet Memes”. In: Mandiberg, Michael (ed.): The Social Media Reader. New York, University Press: 120-134.

Dawkins, Richard (1978): Das egoistische Gen. Heidelberg: Springer.

Dewey, Caitlin (2015): "Russia just made a ton of Internet memes illegal". Washington Post April 10, 2015. washingtonpost.com/news/the-intersect/wp/2015/04/10/russia-just-made-aton-of-internet-memes-illegal. [02.03.2020]

Diekmanshenke, Hajo (2011): „'Schlagbilder'. Diskursanalyse politischer Schlüsselbilder“. In: Diekmannshenke, Hajo/Klemm, Michael/Stöckl, Hartmut (eds.): Bildlinguistik. Theorien Methoden - Fallbeispiele. Berlin, Erich Schmidt: 161-184. (= Philologische Studien und Quellen 228).

Doelker, Christian (2006): Ein Bild ist mehr als ein Bild. Visuelle Kompetenz in der MultimediaGesellschaft. Stuttgart: Klett-Cotta.

Dynel, Marta (2016): "I Has Seen Image Macros!”. Advice Animal Memes as Visual-Verbal Jokes". International Journal of Communication 10: 660-688.

Gervais, Ricky (2013): Twitter is like being able to read every toilet wall in the world. twitter.com/rickygervais/status/1209162673713303552 [03.03.2020].

Hausendorf, Heiko (2009): „Kleine Texte - über Randerscheinungen von Textualität“. Germanistik in der Schweiz - Onlinezeitschrift der Schweizerischen Akademischen Gesellschaft für Germanistik 6: 5-19. sagg-zeitschrift.unibe.ch/6_09/hausendorf.html. [02.03.2020].

Heringer, Hans Jürgen (2017): Interkulturelle Kommunikation. Stuttgart: UTB. (= UTB 2550).

Herwig, Jana (2010): „Fluktuierende Kollektive, lebendiges Archiv: semiologische Praktiken im Imageboard 4chan“. In: Ehardt, Christine et al. (eds.): Inszenierung von Weiblichkeit: Zu Konstruktion von Körperbildern in der Kunst. Wien, Löcker: 1-16.

Hindelang, Götz (2010): Einführung in die Sprechakttheorie. Sprechakte, Äußerungsformen, Sprechaktsequenzen. Berlin: de Gruyter. (= Germanistische Arbeitshefte 27). 
Johann, Michael/Bülow, Lars (2018): „Die Verbreitung von Internet-Memes: empirische Befunde zur Diffusion von Bild-Sprache-Texten in den sozialen Medien“.kommunikation@gesellschaft 19: 1-24.

Johann, Michael/Bülow, Lars (2019): „Politische Internet-Memes: Erschließung eines interdisziplinären Forschungsfeldes“. In: Bülow, Lars/Johann, Michael (eds.): Politische InternetMemes - Theoretische Herausforderungen und empirische Befunde. Berlin, Frank \& Timme: $13-40$.

Kappas, Arvid/Müller, Marion G. (2006): „Bild und Emotion — ein neues Forschungsfeld. Theoretische Ansätze aus Emotionspsychologie, Bildwissenschaft und visueller Kommunikationsforschung". Publizistik 1/51:3-23.

Keller, Rudi (2003): Sprachwandel. Von der unsichtbaren Hand in der Sprache. Tübingen etc.: Francke. (= UTB für Wissenschaft Uni-Taschenbücher 1567).

Klemm, Michael (2002): „Ausgangspunkte: Jedem seinen Textbegriff? Textdefinitionen im Vergleich“. In: Fix, Ulla/Adamzik, Kirsten/Antos, Gerd (eds.): Brauchen wir einen neuen Textbegriff? Antworten auf eine Preisfrage. Frankfurt am Main, Lang: 17-29.

Klemm, Michael/Stöckl, Hartmut (2011): „'Bildlinguistik' - Standortbestimmung, Überblick, Forschungsdesiderate“. In: Diekmannshenke, Hajo/Klemm, Michael/Stöckl, Hartmut (eds.): Bildlinguistik. Theorien - Methoden - Fallbeispiele. Berlin, Erich Schmidt: 7-18. (= Philologische Studien und Quellen 228).

Krämer, Sybille (2009): Gibt es eine Performanz des Bildlichen? Reflexionen über „Blickakte“. userpage.fu-berlin.de/ sybkram/media/downloads/Performanz_des_Bildlichen.pdf. [02.03.2020].

Krieg-Holz, Ulrike/Bülow, Lars (2019): „Internet-Memes: Praktik oder Textsorte“. In: Bülow, Lars/Johann, Michael (eds.): Politische Internet-Memes - Theoretische Herausforderungen und empirische Befunde. Berlin, Frank \& Timme: 89-113.

Lauschke, Marion (2013): Bildakt-Theorie. gib.uni-tuebingen.de/netzwerk/glossar/index.php? title $=$ Bildakt-Theorie.

Leavitt, Alex (2014): „From \#FollowFriday to YOLO: Exploring the Cultural Salience of Twitter Memes". In: Weller, Katrin et al. (eds.): Twitter and Society. New York etc., Lang: $137-154$.

Löber, Nils (2011): In den Unterwelten des Web 2.0. Ethnographie eines Imageboards. TOBIAS-lib.

Maiwald, Klaus (2004): „Das Leben ist voller Überraschungen ...” - Grundschulkinder beobachten Fernsehwerbung. mediendidaktik-deutsch.ewf.uni-erlangen.de/learnweb.data/ medid/dokumente/maiwald2004.pdf. [02.03.2020].

Marx, Konstanze/Weidacher, Georg (2014): Internetlinguistik. Ein Lehr- und Arbeitsbuch. Tübingen: Narr.

Merten, Marie-Luis/Bülow, Lars (2019): „Zur politischen Internet-Meme-Praxis: Bild-SpracheTexte kognitiv-funktional“". In: Bülow, Lars/Johann, Michael (eds.): Politische InternetMemes - Theoretische Herausforderungen und empirische Befunde. Berlin, Frank \& Timme: 195-227.

Miltner, Kate (2011): SRSLY PHENOMENAL: AN INVESTIGATION INTO THE APPEAL OF LOLCATS. London School of Economics and Political Science, Dissertation. 
Morger, Fabia (2017): Memes gegen Rechts. Eine medienlinguistische Analyse von Memes im politischen Diskurs der Durchsetzungsinitiative. su.diva-portal.org/smash/get/diva2: 1164103/FULLTEXT01.pdf. [02.03.2020].

Opletalová, Veronika (2016): „Bildakt, Bildkritik und Bildlinguistik. Der pictorial turn aus fachsprachentheoretischer Sicht". Brünner Beiträge zur Germanistik und Nordistik 2/30: 19-35. Osterroth, Andreas (2015): „Das Internet-Meme als Sprache-Bild-Text“. IMAGE. Zeitschrift für interdisziplinäre Bildwissenschaft. 7/22.

Osterroth, Andreas (2019): „How to do things with memes? - Internet Memes als multimodale Sprechakte". In: Johann, Michael/Bülow, Lars (eds.): Politische Internet-Memes - Theoretische Herausforderungen und empirische Befunde. Berlin, Frank \& Timme: 41-60.

Pauliks, Kevin (2019): „Über kleine Hände und große Affen. Die serielle Narrativität politischer Internet-Memes am Beispiel von Donald Trump“. In: Bülow, Lars/Johann, Michael (eds.): Politische Internet-Memes - Theoretische Herausforderungen und empirische Befunde. Berlin, Frank \& Timme: 61-88.

Sachs-Hombach, Klaus (2003): Das Bild als kommunikatives Medium. Elemente einer kommunikativen Bildwissenschaft. Köln: Halem.

Sachs-Hombach, Klaus (2011): „Bildakttheorie. Antworten auf die Differenz von Präsenz und Entzug“،. In: Stoellger, Philipp/Klie, Thomas (eds.): Präsenz im Entzug. Ambivalenzen des Bildes. Tübingen, Mohr Siebeck: 57-82.

Sandig, Barbara (2000a): „Text als prototypisches Konzept“. In: Mangasser-Wahl, Martina (ed.): Prototypentheorie in der Linguistik. Anwendungsbeispiele-Methodenreflexion-Perspektiven. unter Mitarbeit von Ulla Bohnes. Tübingen, Stauffenburg: 93-112.

Sandig, Barbara (2000b): „Textmerkmale und Sprache-Bild-Texte“. In: Fix, Ulla/Wellmann, Hans (eds.): Bild im Text - Text und Bild. Heidelberg, Winter: 3-30.

Scheiber, Marcus (2019): „Strategien der Verwendung von Bild-Sprache-Gefügen in der politischen Kommunikation“. In: Bülow, Lars/Johann, Michael (eds.): Politische Internet-Memes - Theoretische Herausforderungen und empirische Befunde. Berlin, Frank \& Timme: 143-166.

Schmitz, Ulrich (2007): „Bildakte? How to do things with pictures“. Zeitschrift für germanistische Linguistik 3/35: 419-433.

Schwarz-Friesel, Monika (2009): „Ironie als indirekter expressiver Sprechakt: Zur Funktion emotionsbasierter Implikaturen bei kognitiver Simulation“. In: Bachmann-Stein, Andrea/ Merten, Stephan/Roth, Christine (eds.): Perspektiven auf Wort, Satz und Text. Semantisierungsprozesse auf unterschiedlichen Ebenen des Sprachsystems. Festschrift für Inge Pohl. Trier, Wissenschaftlicher Verlag Trier: 223-232.

Searle, John R. (1965): „What is a speech act?”. In: Black, Max (ed.): Philosophy in America. London, Unwin Hyman: 221-239.

Searle, John R. (1982): Ausdruck und Bedeutung: Untersuchungen zur Sprechakttheorie. Berlin: Suhrkamp.

Searle, John R. (1984): Sprechakte. Ein sprachphilosophischer Essay. Frankfurt: Suhrkamp.

Shifman, Limor (2014): Meme. Kunst, Kultur und Politik im digitalen Zeitalter. Berlin: Suhrkamp.

Siegel, Tanja (2018): “Disney's 'Christopher Robin' Won't Get China Release Amid Pooh Crackdown”. The Hollywood Reporter August 03, 2018. hollywoodreporter.com/heat- 
vision/christopher-robin-refused-china-release-winnie-pooh-crackdown-1131907.

[02.03.2020].

Spitzmüller, Jürgen/Warnke, Ingo H.: Diskurslinguistik. Eine Einführung in Theorien und Methoden der transtextuellen Sprachanalyse. Berlin/New York: de Gruyter.

Staffeldt, Sven (2008): Einführung in die Sprechakttheorie. Ein Leitfaden für den akademischen Unterricht. Tübingen: Stauffenburg. (= Stauffenburg Einführungen 19).

Stalnaker, Robert (2014): Context. Oxford: Oxford University Press.

Stöckl, Hartmut (2004): Die Sprache im Bild - Das Bild in der Sprache. Zur Verknüpfung von Sprache und Bild im massenmedialen Text Konzepte. Theorien. Analysemethoden. Berlin/New York: de Gruyter. (= Linguistik-Impulse \& Tendenzen 3).

Stöck1, Hartmut (2011): „Sprache-Bild-Texte lesen. Bausteine zur Methodik einer Grundkompetenz“. In: Diekmannshenke, Hajo/Klemm, Michael/Stöckl, Hartmut (eds.): Bildlinguistik. Theorien - Methoden - Fallbeispiele. Berlin: Erich Schmidt: 45-70.

Stukenbrook, Anja (2013): „Sprachliche Interaktion“. In: Auer, Peter (ed.): Sprachwissenschaft. Grammatik - Interaktion - Kognition. Stuttgart: Metzler: 217-259.

Thelwall, Mike (2014): „Sentiment Analysis and Time Series with Twitter". In: Weller, Katrin et al. (eds.): Twitter and Society. New York: 83-96.

Vorderer, Peter (2015): „Der mediatisierte Lebenswandel“. Publizistik 3/60: 259-276.

Waseem, Zeerak (2016): “Are You a Racist or Am I Seeing Things? Annotator Influence on Hate Speech Detection on Twitter". Proceedings of 2016 EMNLP Workshop on Natural Language Processing and Computational Social Science: 139-142.

Weidacher, Georg (2019): „Sarkastische Internet-Memes im Flüchtlingsdiskurs“. In: Bülow, Lars/Johann, Michael (eds.): Politische Internet-Memes - Theoretische Herausforderungen und empirische Befunde. Berlin: Frank \& Timme: 167-193.

Wenger, Etienne (2000): Communities of Practice. New York: Cambridge University Press. Zappavigna, Michele (2012): Discourse of Twitter and Social Media: How We Use Language to Create Affiliation on the Web. London/New York: Bloomsbury.

\section{Abbildungsverzeichnis}

Abbildung 1a: img-9gag-fun.9cache.com/photo/ayodx3r_460swp.webp [19.02.2020]. Abbildung 1b: img-9gag-fun.9cache.com/photo/azljqbj_460swp.webp [19.02.2020]. Abbildung 1c: img-9gag-fun.9cache.com/photo/aR0AmP2_460swp.webp [19.02.2020]. Abbildung 1d: img-9gag-fun.9cache.com/photo/a9R7WzZ_460swp.webp [19.02.2020]. Abbildung 2: media.makeameme.org/created/escapes-a-burning-8pagns.jpg [19.02.2020]. Abbildung 3: 66.media.tumblr.com/a4e11d474aa6e3553b578247fe2e8175/tumblr nzw6wffhwX1qe11kdo1_400.jpg [19.02.2020].

Abbildung 4: twitter.com/realDonaldTrump/status/1098218016255414272 [19.02.2020]. Abbildung 5: twitter.com/realDonaldTrump/status/1114631027551375360 [19.02.2020]. Abbildung 6: twitter.com/realDonaldTrump/status/1115057524770844672 [19.02.2020]. Abbildung 7: twitter.com/realDonaldTrump/status/1169782689257447424 [19.02.2020]. Abbildung 8: twitter.com/Irishyrock/status/1169783023769989120/photo/1 [19.02.2020]. Abbildung 9: twitter.com/jovicat/status/1114987325962874885 [19.02.2020]. Abbildung 10: twitter.com/i/status/1169824126606819328 [19.02.2020]. Abbildung 11: twitter.com/cadburybunny/status/1115033074297901056 [19.02.2020]. 
Abbildung 12: twitter.com/BperkBecky/status/1114938652843433986 [19.02.2020]. Abbildung 13: twitter.com/janice987/status/1115222817916821504 [19.02.2020]. 